\title{
Nach-Krieg. Reflexionen in der Kunstkritik der Weimarer Republik am Beispiel des Verismus
}

\section{Olaf Peters}

Martin-Luther-Universität Halle-Wittenberg

\section{Abstract}

Die Aufarbeitung des Ersten Weltkriegs ist ein zentrales Motiv in der frühen Kunst der Weimarer Republik. Dadaismus und Verismus haben dazu bedeutende Beiträge geleistet. Der Aufsatz untersucht und dokumentiert die kunstkritische Auseinandersetzung mit Werken von Max Beckmann, Otto Dix und Georg Scholz. Diese Künstler wurden teilweise unter dem Begriff eines linken Flügels der Neuen Sachlichkeit subsumiert und als Veristen bezeichnet. Ihre mitunter schonungslosen Werke versuchten den Militarismus des Kaiserreichs zu überwinden.

Les réflexions sur la Première Guerre mondiale constituent un motif majeur de l'art dans les premières années de la République de Weimar. Dadaïsme et vérisme ont joué un rôle de premier plan. Le présent article propose des analyses et des informations sur des artistes tels que Max Beckmann, Otto Dix et Georg Scholz. Ces artistes étaient globalement vus comme des représentants de l'aile gauche de la Nouvelle Objectivité et qualifiés de véristes. Leurs œuvres souvent d'une grande brutalité ont essayé de vaincre le militarisme de l'empire wilhelminien.

The reflection of the events and experiences of World War I is one of the main aspects of modern German art at the beginning of the Weimar Republic. Dadaism and Verism made great contributions in this respect. The text investigates and documents the art critical reception of artists like Max Beckmann, Otto Dix and Georg Scholz. These artists were covered by the term Verism and represented the so-called 'left wing' of the New Objectivity-movement of the 1920s. Their sometimes merciless works of art were an attempt to overcome the militarism of the Wilhelmine empire.

\section{Keywords}

Erster Weltkrieg $\bullet$ Verismus $・$ Neue Sachlichkeit $\bullet$ Kunstkritik $・$ Rezeptionsgeschichte

Première Guerre mondiale $\bullet$ vérisme $\bullet$ Nouvelle Objectivité $\bullet$ critique d'art $\bullet$ histoire de la réception

World War I • Verism • New Objectivity • Art criticism • Reception history

Niederlage, Revolution, Bürgerkrieg, Demobilmachung, Hyperinflation sind einige dramatische Schlagworte, die die Situation nach dem Ersten Weltkrieg in Deutschland charakterisieren. Die verbreitete Rede vom ,Tod des Expressionismus' und die sich anschließende Frage nach einem ,neuen Naturalismus' verdeutlichten die künstlerische Zäsur. Sie kam einem Paradigmenwechsel gleich, auch wenn das Neue noch oftmals im Zeichen des Alten begonnen wurde. Das Staatliche Bauhaus in Weimar kann hier als heute international strahlendes Symbol gelten. Gotisch-expressiv, den neuen künstlerischen wie gesellschaftlichen Bau anstrebend, trat es 1919 in der Klassik-Stadt Weimar an die Öffentlichkeit dort, wo man an Goethe und Schiller erinnert und die erste deutsche Demokratie konzipiert wurde. Doch schon 1923 proklamierte das Bauhaus eine neue Einheit aus Kunst und Technik und vollzog so die Zuwendung zur Zeitströmung der
Neuen Sachlichkeit. Diese war schon früher erkannt worden, verdichtete sich jedoch jetzt und wurde ab 1925 nach einer weithin beachteten Ausstellung in Mannheim zum Schlagwort einer ganzen Epoche erklärt, auch wenn die Ausstellung sich auf die Malerei konzentriert hatte.

Das Schlagwort wurde immer ambivalent aufgefasst und blieb umstritten, denn es beinhaltete eine fundamentale Duplizität, die für das Nachfolgende einen Ausgangspunkt darstellt. Man unterschied mit Blick auf die Neue Sachlichkeit von vornherein einen linken und einen rechten Flügel, die man respektive als Verismus und als Klassizismus bezeichnete. In diesem Beitrag geht es um den Verismus und seine kunstkritische Reflexion als zentrale künstlerische Antwort auf den verlorenen Weltkrieg. Eine kritische Darstellung des Verismus und seiner Rezeption bis heute erscheint, trotz einzelner Ansätze, weiterhin als Desiderat der Forschung. Dabei 
wären als Themenfelder zu berücksichtigen: die unmittelbare künstlerische Reaktion auf die Erschütterung durch die Niederlage und die deutsche Revolution von 1918/19; das enge Verhältnis von Verismus und Dadaismus; das Versiegen der Strömung seit Beginn der Stabilisierungsphase der Republik; der vernichtende Hass der Nationalsozialisten auf den Verismus; die höchst selektive, in weiten Teilen negative Rezeption durch die DDR-Kunstgeschichtsschreibung; die späte, oft einseitig politische Rezeption in der Bundesrepublik und schließlich die heutige Strahlkraft der Richtung angesichts der krisenhaft beginnenden 2020er-Jahre. Die nachstehenden Bemerkungen zielen auf einen eng gefassten Teilaspekt, der vor allem die kunstkritische Perspektive aufnimmt.

1919 veröffentliche der spätere Mannheimer Museumsdirektor Gustav Friedrich Hartlaub sein Buch Kunst und Religion, in dem er die Frage nach der Möglichkeit einer neuen religiösen Kunst umkreiste. Zuvor hatte es in Mannheim dazu eine Ausstellung gegeben. Nur zu verständlich erschien der Versuch, Schock und Krise mit einer Hinwendung zur Religion zu verarbeiten, gar zu meistern. Emil Nolde und Max Beckmann waren Hartlaub die wegweisenden Figuren (Abb. 1) und Beckmann wurde ihm in den nächsten Jahren gar zur überragenden Schlüsselfigur einer neusachlichen Malerei. 1919 hielt er zu Beckmanns neuen religiösen Bildern fest:

Die Werke sind außerordentlich und stehen in der gegenwärtigen Produktion einsam da. Es zeigt sich hier, welche Früchte diese opfervolle formale Askese, diese Disziplinierung, der sich der Künstler wohl unter den Eindrücken des Krieges unterworfen haben mag und die inn einen gewaltigen Phantasiereichtum auf wenige knappe figürliche Formeln zusammendrängen lehrt, gezeigt hat. (Hartlaub 1919: 84)

Die ganze neue Produktion des Malers Beckmann wird unmittelbar mit der Kriegserfahrung und einer neuen "gnostisch-religiöse(n)“ Haltung zusammengebracht, so auch das für den Künstler nicht zu bewältigende und nie fertiggestellte Riesenformat der Auferstehung: „Ein sehr strenger Schicksalsernst hat in dem Künstler während der Kriegszeit die Gestaltung des ungeheuren eschatologischen Symbols der Totenauferstehung reifen lassen." (ebd.) Beckmann besaß und verkörperte für Hartlaub eine „tief pessimistische Sachlichkeit" (ebd.: 83), mit der er sich selbst zu erlösen wisse. Der Künstler selbst sprach in der Zeit bekenntnishaft von einer transzendentalen Sachlichkeit und von dem Wunsch, den tief verstörten und enttäuschten Menschen mit seinen Bildern eine neue Kirche zu bauen (vgl. Beckmann 1990 [1918]). Mit diesen Werken und den verwandten Graphiken der Zeit wurde Beckmann zu einem der führenden Veristen und fand gezielt Anschluss an die avantgardistischen Tendenzen seiner Zeit, wie sie 1925 in dem Buch Die Kunstismen dokumentiert wurden, wobei Otto Dix und George Grosz exemplarisch aufgeführt wurden.

Beckmanns künstlerische Wendung sollte auch der Kunsthistoriker Carl Einstein Jahre später mit dem Krieg in einen direkten Zusammenhang bringen, wenn er in seiner Propyläen-Kunstgeschichte von 1926 im Abschnitt über Beckmann schrieb:

Der Krieg kam; endlich entdeckten einige Maler Physiognomie der Zeit; sie hatte sich gründlich dekouvriert, [...]. Man war gepackt und wehrte Erschütterung, die immer unerheblicher blieb als der Anlaß, durch Beobachten ab. Aus Erregung eckte allmählich Gestaltung. [...] Einzige Verteidigung: bis zum Erbrechen feststellen [...]. (Einstein 1926: 154)

Mit dem Begriff der Physiognomie spielte Einstein auf Wilhelm Fraengers brillante Einzelanalyse von Beckmanns Hauptwerk Der Traum von 1921 an. Fraenger hatte seine Interpretation im Untertitel als Beitrag zur Physiognomik des Grotesken bezeichnet (Abb. 2) und weitete sie im Rückgriff auf Beckmanns Graphiken der Zeit zu einer umfassenden Auseinandersetzung mit der unmittelbaren Nachkriegszeit:

In solchen Sprengungen der festen Raumeinheit, der Auflösung der Komposition, in der Herabwertung der menschlichen Gestalt zur seelenlosen Larve, drücken sich leidvolle Erschütterungen aus, welche Max Beckmann widererfahren sind. Diese überreizten Formen sind Sinnbild seines eigenen Schreckens, seines Entsetzens über die Entstellungen der Menschenform, wie sie inm täglich auf der Straße begegnen; seiner Empörung über das Martyrium der grausig hingemordeten Revolutionäre; seines Mitleids mit der tristen Not, welche in demütiger Stille in den Kleinbürgerstuben dahinsiecht; seines Abscheus über das feist gemästete Behagen, das sich bei sch(m)etternden Musiken in den Bars vergnügt. (Fraenger 1924: 50)

Fraenger deutete Beckmanns Bestreben schließlich als Ausdruck eines „Gerechtigkeits-Gedankens“, wobei Beckmanns Form der chaotisch-anarchischen Gegenwart eine Art Netz überwarf, mit der sie geordnet, geregelt, zur Räson gebracht werden sollte. Insofern verschränkte der Kritiker den formalen Ausdruck des Künstlers mit der Annahme eines ethischen Strebens (vgl. ebd.: 57).

Wenn der Kritiker und Museumsleiter Paul Ferdinand Schmidt 1924 in der Zeitschrift Das Kunstblatt seinen Text Die deutschen Veristen überschrieb, dann zählte er allerdings Beckmann nicht dazu. Vor allem George Grosz - den er als „Satiriker" bezeichnete - und Otto Dix, Georg Scholz, Rudolf Schlichter und Otto Griebel - die er „Objektivisten“ nannte hob er hier hervor, als formale und inhaltliche Revolutionäre. Die Revolution, die für Schmidt eine europäische war, wurde vom Ersten Weltkrieg ausgelöst. Jetzt transformierten sich die avantgardistischen Strömungen. Der Kubismus wurde als Ausgangspunkt von Schmidt hervorgehoben, wobei die facettierte Malerei sich jetzt teilweise in eine „konstruktive(n) Flächenform" verwandele oder Léger eine neugotischvertikale Kunst mit neuer Monumentalität realisierte. Parallel betonte Schmidt die neuen, streng aufgebauten, „neuklassizistischen Bilder Derains“ (vgl. Schmidt 1924: 367), und damit jenen Zug der Gegenwartsmalerei, den Gustav Friedrich 


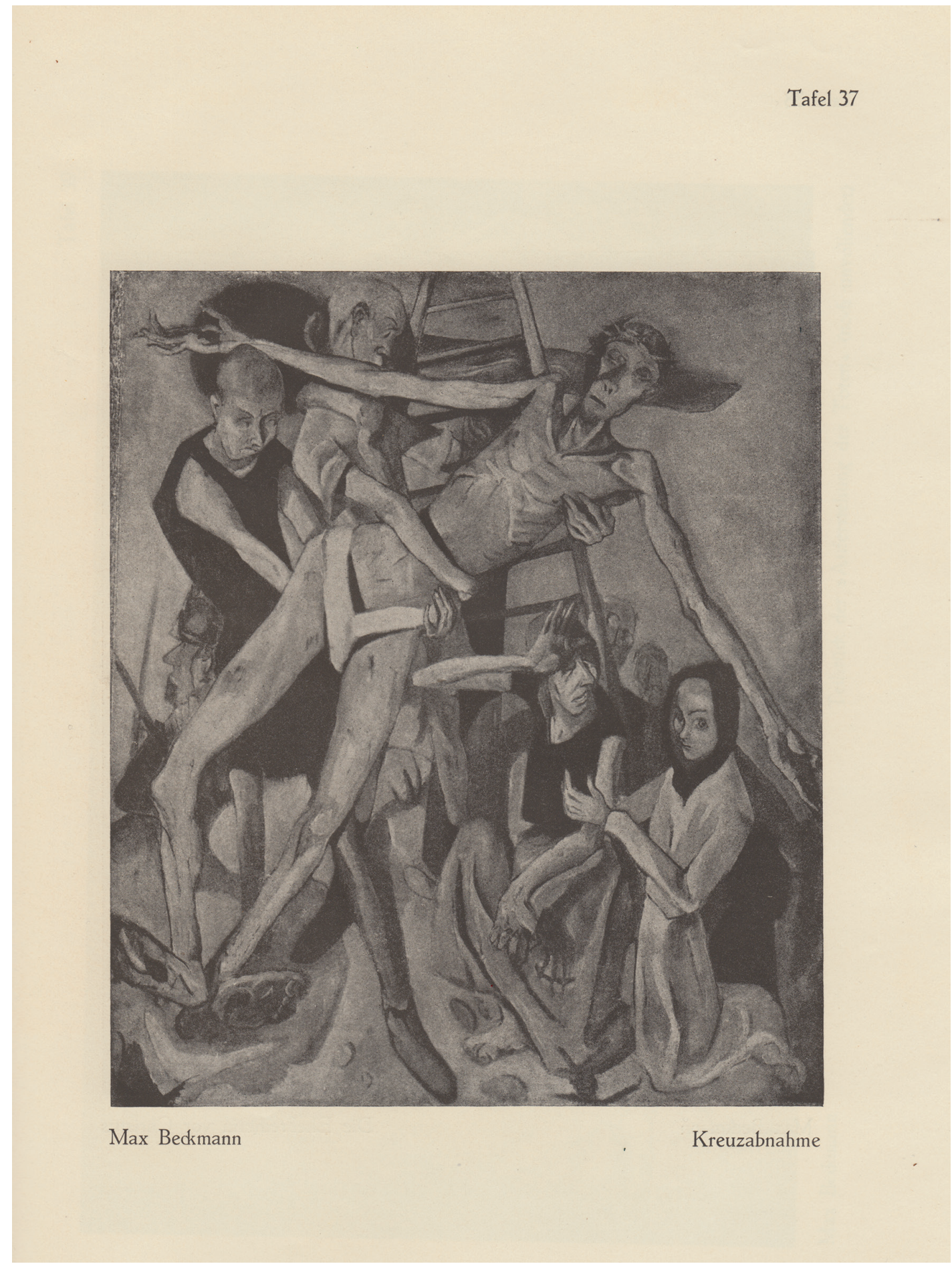

Abb. 1: Max Beckmann, Kreuzabnahme, 1917, in: Hartlaub 1919, Tafel 37

Hartlaub parallel als rechten Flügel der Neuen Sachlichkeit identifiziert hatte.

Die sprachliche Charakterisierung der veristischen Malerei verdient Aufmerksamkeit, weil hier eine Nähe zwischen kunstkritischem Diskurs und Weltkriegserfahrung aufscheint. Schmidt sprach angesichts der Werke von Dix, Griebel und Schlichter von einer „penetrante(n) Genauigkeit der Detailbildung“, von „kalte(r) Leidenschaft“ und von „tote(r) Anschaulichkeit“ (ebd.: 368). Der Blick auf die Maschine, der eine unmenschliche Präzision anhafte, die sich angesichts ihrer Logik bis zu einem Grauen steigern könne, deutete nochmals die Erfahrung des Krieges an, der von beängstigenden Phänomenen und drastischen Konsequenzen - kalte Präzision und groteske Vertotung - geprägt war. Der Krieg selbst wird hier nicht angesprochen, aber Schmidt zählt in eindrucksvoller Manier Begriffe und Wendungen auf, die eine distanzierte Härte beschreiben und so die neusachliche Mentalität der Künstler fassen sollen. So werden die Wörter Bedrohung, 


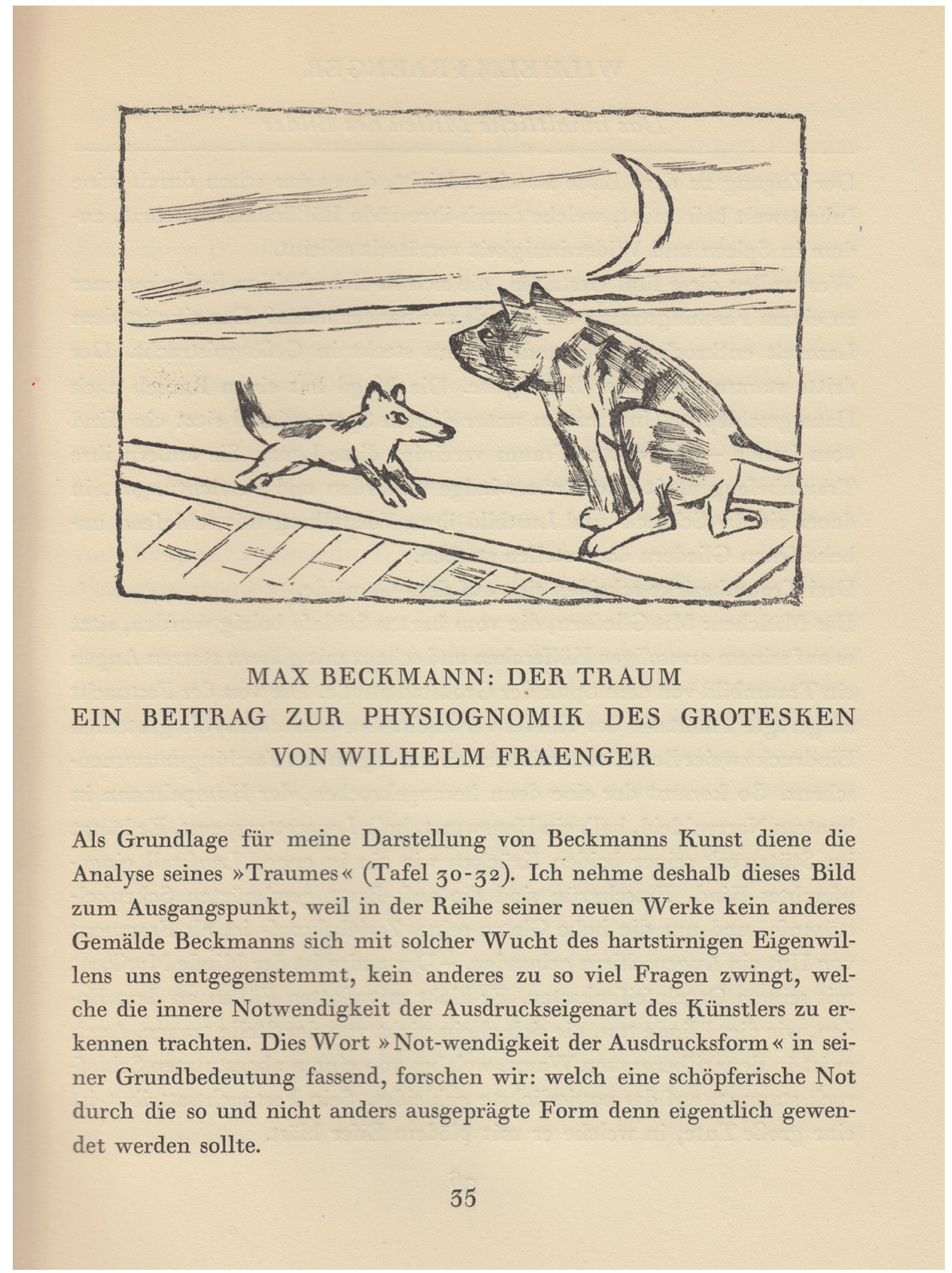

Abb. 2: Titelseite des Aufsatzes von Wilhelm Fraenger, in: Max Beckmann, München 1924, 35

Nacktheit, unerbittliche Tatsächlichkeit, ratlose Erbitterung, Erbarmungslosigkeit, Gottlosigkeit, grelles Scheinwerferlicht in einem Absatz des Textes vom Autor zusammengezogen, um den endgültigen Bruch mit der Vorkriegswelt sprachlich nachdrücklich zu verdeutlichen und die neue Bildästhetik zu charakterisieren. Wörtlich heißt es, so als ob Schützengraben und Niemandsland beschrieben würden:

Diese Objektivität wirkt auf den Durchschnittsmenschen darum so aufstachelnd, weil sie auf Gemüt und Sentimentalität nicht die geringste Rücksicht nimmt; weil sie lustlos ist und die furchtbare Öde einer gottlosen Welt unter grelles Scheinwerferlicht stellt. Der Bürger will immer Romantik und Überzuckern der Tragik, in irgend einer Form; hier aber ist nichts als Absage an alles Romantische, an den letzten Rest von schönem Schein und Trug, der unsere Existenz verklären könnte. (ebd.: 370)
Hinter der neuen Kunst scheint aber für den Kritiker doch eine Hoffnung auf und diese wird in einem Bild des (Nach-) Krieges gefasst: „Man kann sich nicht so grenzenlos an die Dinge verlieren ohne eine Liebe, die verborgen sein mag unter dem Abscheu vor der Gegenwart, die aber stark genug ist, um eines Tages, wenn der Schutt fortgeräumt sein wird, den Aufbau einer neuen Welt aus der Wahrheit zu beginnen." (ebd.: 371) Die Katastrophe des Krieges verlange einen erbarmungslosen und unerschrockenen Blick. Dieser verberge womöglich zugleich eine Liebe, die sich zunächst dem Schutträumen und dann dem Aufbau widmen könne. Anhand von Dix skizzierte Schmidt kurz die noch anfängliche Entwicklung, vom satirisch-scherzhaften Dadaismus zum Verismus der Kriegskrüppel, die 1920 auf der Ersten Internationalen Dada-Messe in Berlin gezeigt und von Schmidt selbst für das Dresdner Stadtmuseum erworben worden waren. 
Die Grenze zwischen Dada und Verismus war für die Zeitgenossen fließend.

Der Karlsruher Verist Georg Scholz nahm ebenfalls an der Ersten Internationalen Dada-Messe in Berlin teil und produzierte in der ersten Hälfte der 1920er-Jahre eine größere Zahl hervorragender Bilder, die nüchterne Präzision und ätzenden Humor vereinigten. Schmidt würdigte das mit den Worten:

Die Vereinigung von blendender Bestimmtheit in Wiedergabe toter Gegenstände wie Maschinen, Zimmern, Dorfstraßen, mit einer gargantuesken Maßlosigkeit im menschlich Fratzenhaften gehört wohl überhaupt zum Stärksten, das deutscher Kunst seit der großen Erneuerung gelungen ist. (ebd.: 372)

1923 erschien ein erster großer Artikel über Scholz im Kunstblatt, den Hans Curjel verfasst hatte. Der Autor stellte die Entwicklung des neuen Naturalismus zweifach motiviert dar: zum einen und zunächst als kunstimmanente Konsequenz einer formalen Entwicklung, die für den sogenannten rechten, klassizistischen Flügel der Neuen Sachlichkeit konstitutiv sei, ohne dass Curjel ihn schon so nennen konnte. Zum anderen gab es die künstlerische Reaktion auf die politischen Umstände der Gegenwart. Dabei kam Scholz ins Spiel, dem der Kritiker Curjel aber eine künstlerische Entwicklung bescheinigte:

\section{Das Erlebnis der Gegenwart brachte um 1920 den Umschwung zur gegenständlichen Malerei. Die polemischen Elemente herrschen vor; aller Sattheit, aller Verbohrtheit, aller herzigen Banausen-Sentimentalität, aller gepfropften Sexualität, aller kapitalistischen Rohheit, aller vaterländischen Dummheit wird unerbittlicher Kampf angesagt, der mit brutaler Offenheit ge- führt wird. (Curjel 1923: 260)}

Bilder wie den Deutschen Kriegerverein hielt der Kritiker für zeitgenössische Bilderpredigten, die „Pestbeulen“ ins Licht rücken, „die der Bürger aufzuschneiden sich scheut“ (ebd.). Aber es sind bei Scholz nur ganz wenige Bilder, die in der unmittelbaren Nachkriegszeit auf einen aus der Zeit gefallenen, anachronistisch wirkenden nostalgischen Militarismus hinwiesen. Und auch in der Kunstkritik wurde bei einem Veristen wie Scholz der zurückliegende Krieg offen kaum thematisiert, auch wenn er als tiefe Zäsur präsent war.

Zudem konnten die Bilder Gefahr laufen, als bloße Sensationsbilder rasch beiseitegelegt zu werden. Der KunstblattHerausgeber Paul Westheim hat das bereits 1922 mit dem Begriff des „arrivierten Öldruck“ zu fassen versucht. In seinem Artikel war Georg Scholz prominent ganzseitig mit der Lithographie Wir Deutschen fürchten Gott, sonst nichts auf der Welt von 1921 abgebildet, die drei Kriegerverein-Mitglieder (darunter schon einen Hakenkreuzler) zeigte (Abb. 3). Dem Verismus wurde dabei der Rückgriff auf fast plakatartige Gegenständlichkeit als Versuch ausgelegt, das Atelier zugunsten der Öffentlichkeit zu verlassen. Der Erfolg eines solchen Versuchs war nach Westheim fraglich, denn die drastische Ästhetik der Veristen könne darin enden, „daß gekitzelte Nerven sich an einer Sensation mehr zu delektieren vermögen“
(Westheim 1922: 348). Früh wurde die Möglichkeit erkannt, dass eine auf den Krieg folgende veristische Skandal- oder Schock-Ästhetik ins Leere laufen könne, da die avisierten Massen entweder desinteressiert oder ästhetisch nicht aufnahmebereit waren.

George Grosz hingegen hielt zu der Zeit noch an der politischen Vision einer klassenkämpferischen Kunst fest, forderte die Solidarisierung mit den ausgebeuteten Schichten und relativierte die Fortschritte einer formalistischen Avantgarde, indem er sie zu, wenn auch durchaus interessanten oder ästhetisch wertvollen „Atelier-Probleme(n)“ herabstufte (Grosz 1921: 11). Die Ästhetik nach dem Ersten Weltkrieg hatte sich bei ihm an einem sozial-technologischen, wirtschaftstechnokratischen Leitbild zu orientierten: „Die Sachlichkeit und Klarheit der Ingenieurzeichnung ist ein besseres Lehrbild als das unkontrollierbare Geschwafel von Kabbala und Metaphysik und Heiligenekstase" (ebd.: 14). Grosz situierte den Verismus zu diesem frühen Zeitpunkt für eine kurze Phase zwischen anti-expressionistischem Dadaismus und fortschrittsoptimistischem Konstruktivismus, womit eine in die Zukunft gewandte Perspektive nach dem Bankrott des Systems eröffnet werden sollte.

„Pleite glotzt euch an restlos“. Unter dieser Überschrift stand ein anonym veröffentlichter Text von Carl Einstein aus dem Jahr 1919 in der Zeitschrift Die Pleite, die bald verboten und von der Zeitschrift Der blutige Ernst abgelöst wurde. Herausgegeben wurde Die Pleite, die auch als publizistisches Organ des politischen Berliner Dadaismus fungierte, von Wieland Herzfelde und George Grosz. Der Text war eine durchgängige Anklage und warf vor allem den Regierungssozialisten einen doppelten Verrat vor: Verrat an den Arbeitern und Verrat an der Revolution. Andere Texte plädierten für die Abschaffung des Besitzes oder geißelten den Militarismus, entwarfen das Bild des „dämlich-stumpfsinnige(n) Schiebers: Er monotont Zweck, Zweck, Zweck" und nahmen Europa nach dem Krieg in den Blick: „Europa, ein Kontinent, der vor lauter Zwecken und Zielen verwest. Man krepiert an der sinnvollen Entwicklung“ (Einstein 1919, 39).

Carl Einstein, der sich mit seiner epochalen Negerplastik von 1915 schon einen Namen gemacht hatte, wandte sich in diesem politischen Kontext einer neuen Kunst zu und verabschiedete eine alte. Ein zentraler Reflex in seiner Kritik war das Ende des Expressionismus und seine Zuwendung betraf den Verismus. Einstein wollte keine Kritiken schreiben, wollte die Kunst nicht beschreiben, hielt er das doch für „ein Geländer der Form- und Stoffarmen." Einzige Rechtfertigung für inn war der „propagandistische Grund“, auch wenn er das Gefühl hatte, damit eher dem Kunsthandel als dem Künstler zu dienen (vgl. Einstein 1920: 59). Eine erste Kritik der Zeit widmete sich dem Karlsruher Zeichner und Graphiker Rudolf Schlichter, der sich wie Scholz zu der Zeit auch mit den Berliner Dadaisten verband und ebenfalls an deren Erster Internationaler Dada-Messe teilnahm. Allerdings drehte sich der Text um eine Rechtfertigung Einsteins, überhaupt Kunstkritik zu betreiben. Der Anti-Expressionismus der Veristen wurde hervorgehoben und der Verismus als stofflicher Realismus an die Seite des 


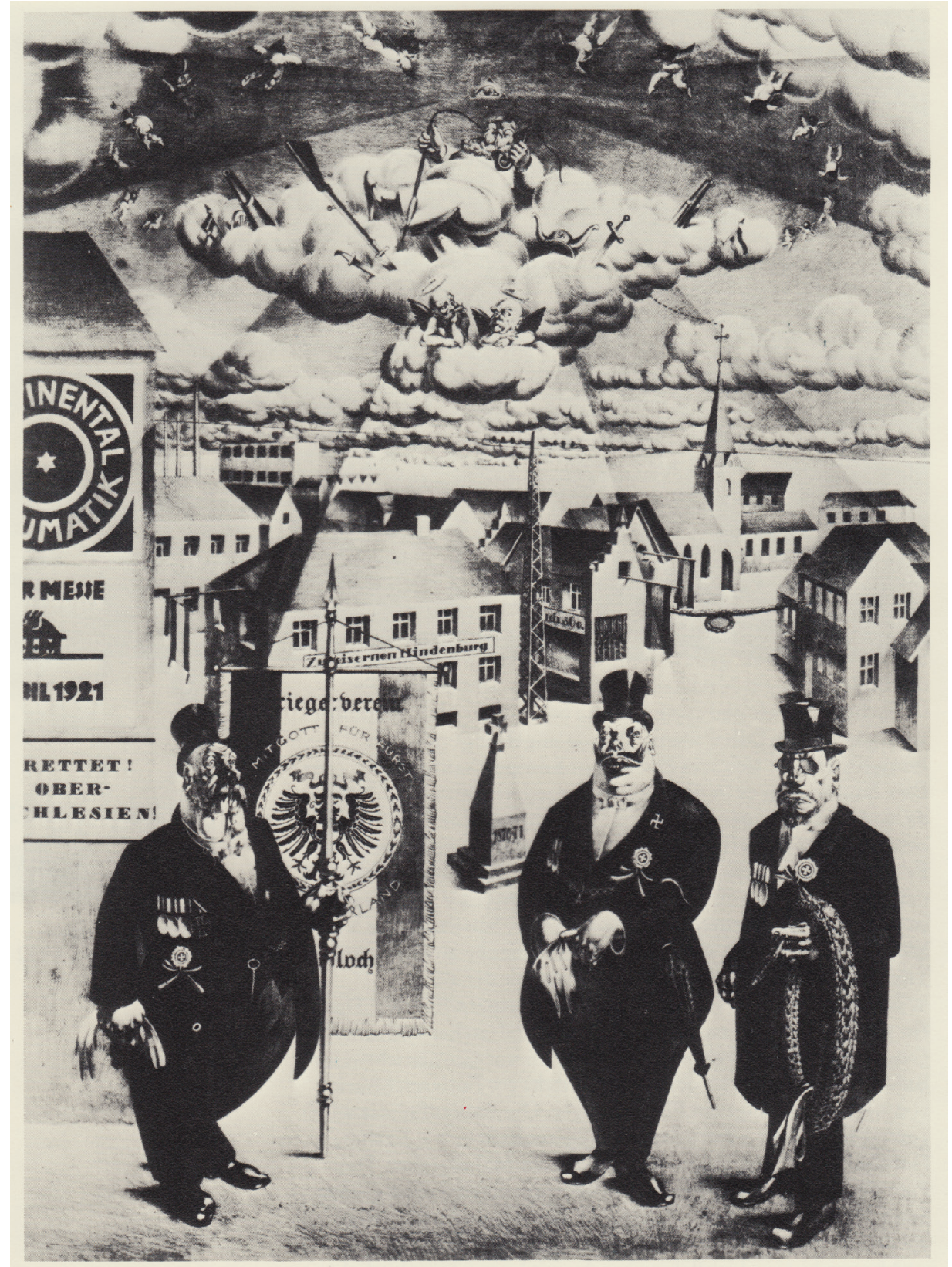

Abb. 3: Georg Scholz: Wir Deutschen fürchten Gott, sonst nichts auf der Welt (Kriegerverein), 1921, in: Das Kunstblatt, Jg. 6, 1922, 345

abstrakten Raumrealismus des Kubismus gestellt; Verismus und Kubismus waren Einstein zwei aktuelle Formen des Realismus.

Otto Dix widmete sich Einstein 1923, ebenfalls im Kunstblatt. Der Text war eine furiose Beschreibung des Verismus und pointierte schon ganz am Anfang: „Malerei, ein Mittel kühler Hinrichtung" (Einstein 1923: 264). Auf den Krieg ging dieser Text aber ebenfalls nicht ein. In der drei Jahre später erschienenen Propyläen-Kunstgeschichte machte Einstein den Bezug allerdings klar:

Dix ist der Sohn des Krieges und vergeblicher Revolte, entschlossen, nicht allzu rasch zu vergessen; er wagt zeitsachlichen Kitsch, doch Malerei kann sich daran selbst leicht banal erweisen; man vertraut zu sehr erregtem Motiv. 1924 versuch- te er das Zeichen des Krieges zu malen - peinliche Allegorie. (Einstein 1926: 156)

Einsteins Kritik scheint aus heutiger Sicht durchaus ungerecht und verkürzt. Der am Kubismus und an der afrikanischen Kunst geschulte Kritiker forderte nach dem Verismus, der in der Stabilisierungsphase der Weimarer Republik allmählich Geschichte wurde und zeitkritischer Relevanz eingebüßt hatte, erneut eine stärkere formale Konzentration. Bekannt geworden ist der Schlusssatz des kurzen Abschnitts, der zwar Dix ' inhaltliche Ausrichtung anerkannte, die künstlerische Umsetzung der Themen aber kritisierte: „Vielleicht ist man im Herzen malender Reaktionär am linken Motiv" (ebd.: 157). So machte Einstein einen Widerstreit zwischen Intention und Form aus, hätte doch auch die Form dem revolutionären 
Inhalt zu folgen, adäquat zu sein. Einsteins Blick war formal an der französischen Moderne geschult und verfehlt fast notwendig den malerischen Ansatz der Veristen, mit denen er inhaltlich sympathisierte. Der Kritiker Paul Fechter hingegen beschrieb am Beispiel des dadaistisch-veristischen Gemäldes Barrikade (Abb. 4) den Ansatz von Dix in Abgrenzung zum damals erneut diskutierten Naturalismus mit diesen Worten:

Daneben braucht man nur einmal an das Dixsche Barrikadenbild zu denken. Da war die Todeswunde eines Matrosen als großes, schwarzes Loch in die Leinwand geschlagen, und die Wundränder mit dicken Farbwülsten wie von geronnenem Blut umklebt. Hier wurde die neue Absicht, der tendenziöse Naturalismus, den man nicht ganz zu Unrecht als Verismus bezeichnet hat, noch deutlicher sichtbar. Der Abstand von der menschlichen Wirklichkeit, die allein als Natur empfunden wird, soll auf ein Minimum reduziert werden. Aber nicht, weil man sachlich sein will, wie die alten, sondern weil ein Fanatismus des Darstellen-, des Aufzeigenwollens dahintersteht. (Fechter 1923: 324)

Fechter besaß das Gespür, den durchaus experimentellen und möglicherweise auch effekthascherischen Formwillen von Dix zu erkennen, der eben nicht nur mit seinen Inhalten, sondern mit seinem Gestaltungsvermögen irritierte. Die Drastik einer hier beschrieben willentlichen physischen Beschädigung der Leinwand - da das Original verloren ist, lässt sich das nicht mehr anschaulich nachvollziehen - in Kombination mit einer haptisch-visuellen Erfahrung von scheinbar geronnenem Blut scheint vor allem über Dix ungewöhnlich direkte Kriegserfahrung erklärbar, da der Maler vier Jahre an der Front gestanden hatte. Dem Kritiker Julius Meier-Graefe, der ein exponierter Verehrer französischer Malerei war, mussten diese und ähnliche Werke als ästhetischer Affront erscheinen, negierten Dadaismus und Verismus doch traditionelle bürgerliche Kunstvorstellungen. Diese vermeintliche Negation, die zugleich einen Neubeginn oder ein Wiederanknüpfen an andere Traditionen bedeutete, muss als Reaktion auf den Krieg und als Bruch mit tradierten Werten verstanden werden.

Der bereits erwähnte Paul Westheim beschäftigte sich 1926 - dem Jahr einer großen Gemälde-Ausstellung von Dix in der Berliner Galerie Neumann-Nierendorf - ebenfalls mit dem Künstler und brachte dabei die an den Krieg geknüpfte Erfahrung der Veristen und ihrer schonungslosen Realitätszuwendung auf einen Punkt, wobei er aber die Form wieder vernachlässigte: „In Gleichnissen zu reden, auch das haben sie seit 1914 gelernt, hat keinen Sinn mehr; damit ist zu skrupellos und unverschämt geschwindelt worden. Nur eins kann noch überzeugen, kann bessern helfen: die Tatsache, die nackte, brutalnackte Tatsache" (Westheim 1926: 143). Westheim bringt das mit deutlichen, ja schonungslosen Worten zum Ausdruck: „Bei Dix entbreitet sich das Pandämonium aller durch Kriegs- und Nachkriegsnöte entfesselten Untermenschlichkeiten. Er ist der rabiat gewordene Sachse, bei dem im Schützengraben, in Blutschlamm und Gasschwaden - die Gemütlichkeit aufgehört hat" (ebd.).
Bei diesem und einem zweiten Text über Dix, der wenig später in derselben Zeitschrift erschien, ist mit Blick auf die Auseinandersetzung mit dem Krieg das neu bedachte Verhältnis von Krieg, Werk und Wertung wichtig. Gerade den Kriegsdarstellungen von Dix bescheinigte Westheim einen „intransiganten ,Realismus'“, von dem er annahm, dass er sich nicht nur einer Kunstauffassung verdanke. Im Hintergrund stünde unausgesprochen eine neue Auffassung von Wirklichkeit und Gegenwart überhaupt.

Wichtig ist Westheims Hinweis auf eine Haltung des Malers:

Unter der Maske, wenn man das sagen darf, ungeschminktester und unerbittlichster Wahrhaftigkeit wird hier offenbar ein Ethos, das umso stärker, aufreizender wirkt, als Dix nicht wieder mit aufgehobenem Finger predigt, sondern scheinbar uninteressiert, scheinbar ohne Folgerung daraus zu ziehen, sachlich feststellt, was und wie es gewesen ist. (ebd.: 144)

Westheim paraphrasierte hier Leopold von Rankes berühmtes historistisches Diktum und wandte es auf den Verismus an; eine Unmittelbarkeit zu Gott war freilich nicht mehr gegeben, denn der war dem Nietzschaner Dix endgültig gestorben und lag in Verkörperung des Sohnes mit einer Stacheldraht-Krone umgedreht im Blutschlamm. Das Heilsgeschehen wurde durch die Kriegserfahrung invertiert. Daneben wäre wie bei der Barrikade der materielle Aspekt des Gemäldes Schützengraben hervorzuheben, der einigen Kritikern als übertrieben erschien (Abb. 5). Dix‘ Position wurde anerkennend, aber auch ambivalent beurteilt, was wohl auch damit zusammenhing, dass sein vielschichtiges Werk nicht leicht einzuordnen war. Auch wurde die Gegenwart selbst als Zwischenperiode wahrgenommen und war eine weitere Entwicklung für die Zeitgenossen noch nicht absehbar. Dennoch wurde die sachliche Tendenz bei Dix jetzt in den Vordergrund gerückt. Im Katalog der Berliner Ausstellung schrieb erneut Paul Ferdinand Schmidt und stellte Dix als „Maler der Kriegsverneinung" dar, „der in Bildern und 50 furchtbaren Radierungen für das selbst miterlebte Grauen eine unerhörte Form fand." Daran schloss er das Lob an: „Diesen Maler werden die kühlen Vernünftler niemals ergründen, heiß und kalt bläst sein Atem aus dem Munde; er ist der Gestalter unserer Zeit: der Gestalter, den wir brauchen, Künder einer aus den Fugen geratenen Welt" (Schmidt 1926: 7). Und wohl in der Berliner Zeitung schrieb P.F. (Paul Fechter):

Das Krasse, Scheußliche, das aus einem Aufruhr des ganzen Wesens wie eine Naturgewalt hervorbrach, mußte dieser Mensch loswerden, um nicht daran zu ersticken. Es ist so stark, daß es als schreckliches Dokument, von unserer Zweiten Schande' die Zeiten der Zersetzung überdauern wird, wenn es auch auf zartbesaitete Gemüter einen negativen Eindruck macht. (P.F. 1926)

Ein Jahr später sollte Ernst Kállai nochmals im Kunstblatt unter dem funkelnden Titel Dämonie der Satire Dix' Kriegsbild Schützengraben ebenfalls die eindeutige, moralisch-pazifistische Aussage absprechen. Das stellt für prominente 


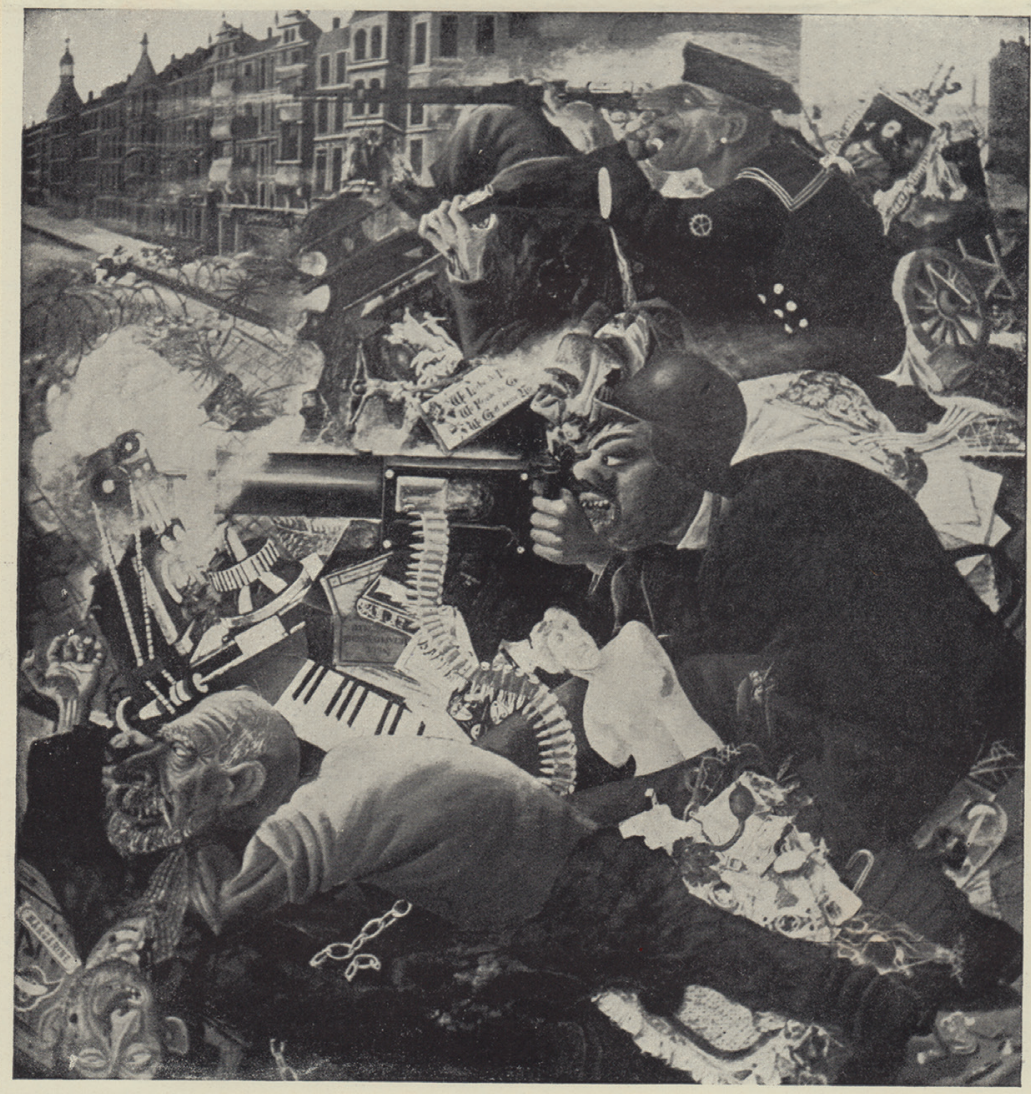

OTTO DIX

Barrikade, Gemälde

Abb. 4: Otto Dix: Barrikade, 1920, in: Neue Blätter für Kunst und Dichtung, Jg. 3, Erster Halbjahrsband, 1920/21, 43

Dix-Forscher wie Dietrich Schubert weiterhin eine Herausforderung dar, kommt der umfassend informierte Kállai doch zu dem ambivalenten und zugleich bedenkenswerten Urteil: „Das Schützengrabenbild von Dix könnte ebensogut der Gegenstand höchster Anbetung eines fanatischen Kriegsgottverehrers, als pazifistisches Propagandamittel sein" (Kállai 1927: 97). Die allgemeine und in der Diffamierung durch die Nationalsozialisten kulminierende Rezeption des Bildes sah in dem Schlüsselwerk eher eine pazifistische Aussage, wenn nicht gar ein Vehikel der defätistischer Wehrkraftzersetzung. Doch entscheidet die mehrheitliche Rezeption eines Werkes nicht über das Ergebnis von dessen formalästhetischer und ikonographischer Analyse und Interpretation.

Der von Kállai vorgenommene Vergleich mit den altdeutschen und romantischen Meistern erklärt den Begriff der Dämonie im Titel, scheint doch hier ein bildlicher Exorzismus vorzuliegen, bei dem allerdings der Teufel mit Beelzebub auszutreiben versucht werde: „Die Abwehr des Abscheulichen wird mit einem pathetischen Zeremoniell der Heraufbeschwörung des Abscheulichen ausgeübt, daß schließlich die Frage ganz und gar offen läßt, ob es sich hier um eine Ablehnung oder um einen Kult handelt" (ebd.: 99). Kállai unterstellt Dix die 


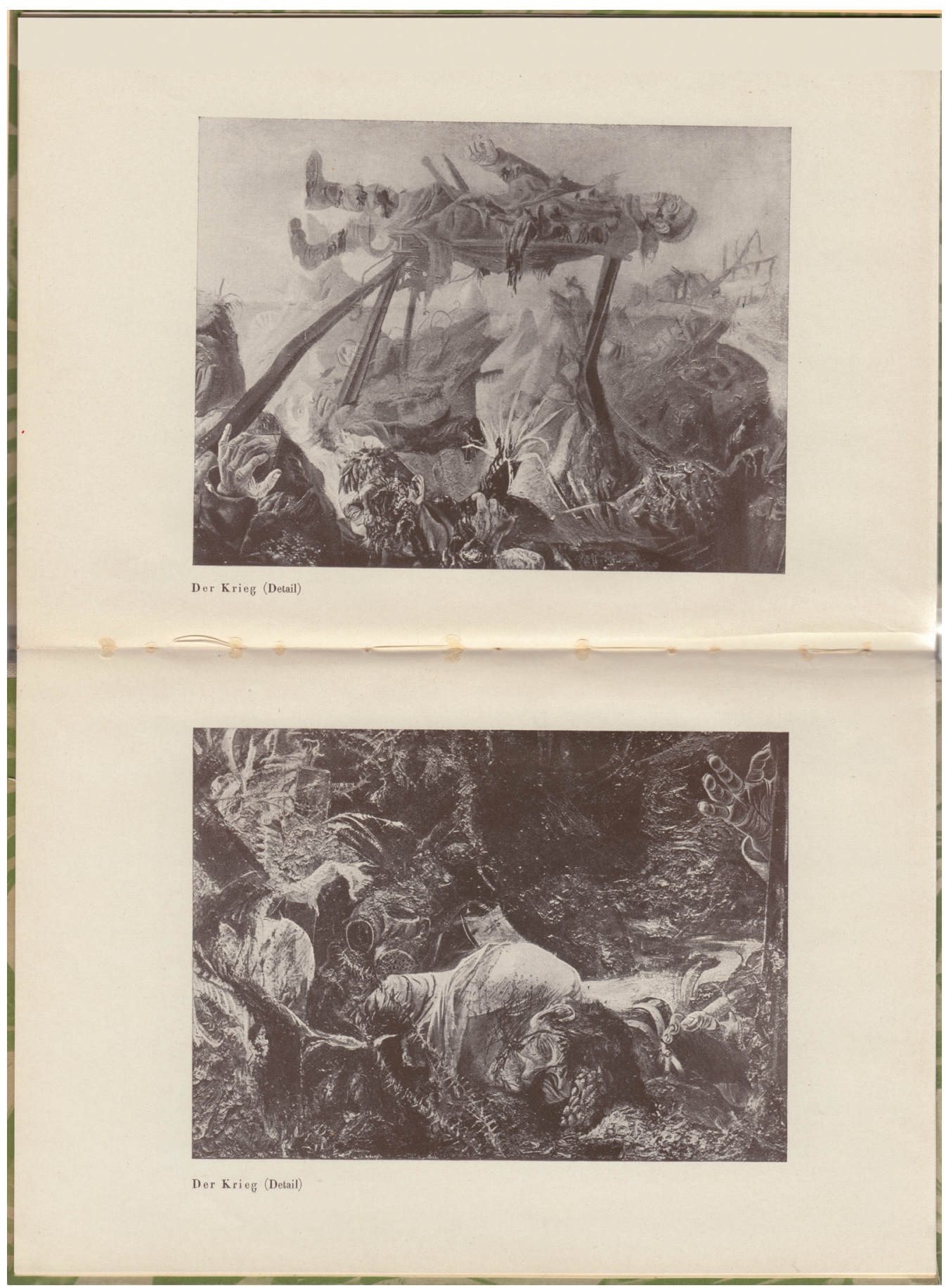

Abb. 5: Doppelseite mit Details von Otto Dix‘ Schützengraben (Der Krieg), in: Wolfradt 1924

Absicht, den Krieg abzuwehren, zu kritisieren, vielleicht sogar zu bekämpfen. Aber die Intensität der Darstellung verrät dem Kritiker, dass der Künstler keine politische Haltung einnehmen könne, sondern im Prozess der Formfindung und -ausführung vollständig von der „Allmacht des Krieges“ überzeugt sei: „Dix glaubt an den Krieg“, sagt Kállai und realisiert damit die von Nietzsche geprägte Weltsicht des Malers, der trotz und aufgrund seiner eigenen Kriegserfahrung nicht zum gesinnungsethischen Pazifisten werden konnte.

Kampf und Leid, Werden und Vergehen waren im heute verlorenen Schützengraben in die monumentale und als
Landschaft konzipierte, erinnernde Bildvision gebannt. Dieses Bild und die darauf reagierende ambivalente, vielleicht auch etwas ratlose Kritik sind die offen perspektivierten Höhepunkte des Nach-Lebens des Krieges mit Blick auf den Verismus. Der Kampf um die Erinnerung setzte ein Jahr nach Kállais bemerkenswertem Artikel ein. Das Jahr 1928 verlangte von Dix zehn Jahre nach der Niederlage eine erneute Entscheidung, sich dem Thema zu stellen. Und wieder entstand mit dem Triptychon Der Krieg ein höchst ambivalentes Bild, das einerseits vor einem neuen Krieg warnen wollte und inn andererseits - gleichsam 
geschichtsphilosophisch aufgeladen - als zwangsläufig wiederkehrend zu interpretieren schien. Die Epoche des Nach-Kriegs hatte sich für die hellsichtigen Zeitgenossen unmerklich zu einem drohenden Zeitalter des Vor-Kriegs gewandelt. Schlafwandeln (Christopher Clark) wurde durch kriegslüsternen Revanchismus ersetzt, der sein Ziel fest im Blick hatte und 1939 auch fand. Paul Fechter hatte 1926 anlässlich der Berliner Dix-Ausstellung von einer „momentane(n) Friedhofsruhe" gesprochen; davon, dass die ,Deutsche Passion“ „noch lange nicht zu Ende“ sei und das Land noch durch ungeahnte wirtschaftliche und politische
Höllen hindurch müsse (P.F. 1926). Er sollte auf schreckliche Weise Recht behalten. Und anders als er vermutete, überdauerten Dix' veristische Bilder nicht als Dokumente einer ,Zeit der Zersetzung', sondern wurden sie von den Nationalsozialisten nicht nur als deren Ausweis, sondern als deren Ursache angesehen und in der Ausstellung und gleichnamigen Broschüre „Entartete Kunst“ diffamiert. Heute müssen viele von ihnen als verloren gelten und können wir den bedeutenden künstlerischen Antworten auf den Krieg nur mittelbar, durch Beschreibungen und Bewertungen von Zeitgenossen nahekommen.

\section{Auswahlbibliographie:}

Ausst.-Kat. Frankfurt/M. (2017), Glanz und Elend in der Weimarer Republik, hrsg. von Ingrid Pfeiffer, Schirn Kunsthalle Frankfurt/M. 2017/18, München, Hirmer.

Ausst.-Kat. Venice/Los Angeles (2015), New Objectivity: Modern German Art in the Weimar Republic 1919-1933, by Stephanie Barron and Sabine Eckmann, Museo Correr, Venice and Los Angeles County Museum of Art 2015/16, Munich et al., Prestel.

Beckmann, Max (1990 [1918]), „Ein Bekenntnis“, ders., Die Realität der Träume in den Bildern. Schriften und Gespräche 1911 bis 1950, hrsg. von Rudolf Pillep, München und Zürich, Piper, 20-23.

Crockett, Dennis (1999), German Post-Expressionism. The Art of the Great Disorder 1918-1924, University Park, Pennsylvania, UP, 145-158.

Curjel, Hans (1923), „Zur Entwicklung des Malers Georg Scholz“, in Das Kunstblatt, Jg. 7, 257-264.

Die Kunstismen (1925), hrsg. von El Lissitzky und Hans Arp, Erlenbach-Zürich, München und Leipzig, Eugen Rentsch.

Einstein, Carl (1981 [1919]), „Freie Bahn dem Tüchtigen“, in Der blutige Ernst, Berlin, Nr. 4, wieder abgedruckt in Einstein (1981), 34-39.

Einstein, Carl (1981 [1920]), „Rudolf Schlichter“, in Das Kunstblatt, 4. Jg., 105ff., wieder abgedruckt in Einstein (1981), 59-61.

Einstein, Carl (1981 [1923]), „Otto Dix“, in Das Kunstblatt, Jg. 7 , 97ff., wieder abgedruckt in Einstein (1981), 264-267.

Einstein, Carl (1926), „Die Kunst des 20. Jahrhunderts“ (= Propyläen-Kunstgeschichte, Bd. XVI), Berlin, Propyläen.

Einstein, Carl (1981), „Werke“, Bd. 2, 1919-1928, hrsg. von Marion Schmid unter Mitarbeit von Henriette Beese und Jens Kwasny, Berlin, Medusa.

Fechter, Paul (1923), „Die nachexpressionistische Situation“, in Das Kunstblatt, Jg. 7, 321-329.

P.F. (Fechter, Paul) (1926), „Otto Dix“, in Berliner Zeitung, Nr. 32
Fleckner, Uwe (2006), Carl Einstein und sein Jahrhundert. Fragmente einer intellektuellen Biographie, Berlin, Akademie, 123-166.

Fraenger, Wilhelm (1924), „Max Beckmann. Der Traum - Ein Beitrag zur Physiognomik des Grotesken“, in Max Beckmann, von Curt Glaser, Julius Meier-Graefe, Wilhelm Fraenger und Wilhelm Hausenstein, München, R. Piper \& Co., 35-58.

Grosz, George (1921), „Zu meinen neuen Bildern“, in Das Kunstblatt, Jg. $5,11-14$.

Hartlaub, Gustav Friedrich (1919), „Kunst und Religion. Ein Versuch über die Möglichkeit neuer religiöser Kunst“, Leipzig, Kurt Wolff.

Kállai, Ernst (1927), „Dämonie der Satire“, in Das Kunstblatt, Jg. 11, 97-104.

Kleinschmidt, Erich (1995), „Von der Schwierigkeit, über Bilder zu schreiben. Zur Poetologie der Kunst in Carl Einsteins Die Kunst des 20. Jahrhunderts", in Jahrbuch zur Literatur der Weimarer Republik, Bd. 1, 1995, 255-277.

Osterkamp, Ernst (1995), „Däubler oder die Farbe - Einstein oder die Form. Bildbeschreibung zwischen Expressionismus und Kubismus", in Boehm, Gottfried / Pfotenhauer, Helmut (Hrsg.), Beschreibungskunst - Kunstbeschreibung. Ekphrasis von der Antike bis zur Gegenwart, München, Fink, 543-568.

Schmidt, Paul Ferdinand (1924), „Die deutschen Veristen“, in Das Kunstblatt, Jg. 8, 367-373.

Schmidt, Paul Ferdinand (1926), „Otto Dix“, in Ausst.-Kat. Otto Dix, Galerie Neumann-Nierendorf, Berlin, 5-7.

Schubert, Dietrich (2013), Künstler im Trommelfeuer des Krieges 1914-18, Heidelberg, Wunderhorn.

Westheim, Paul (1926), „Dix“, in Das Kunstblatt, Jg. 10, 142-146.

Windhöfel, Lutz (1995), Paul Westheim und Das Kunstblatt. Eine Zeitschrift und ihr Herausgeber in der Weimarer Republik, Köln, Weimar und Wien, Böhlau.

Wolfradt, Willi (1924), „Otto Dix“, Leipzig, Klinkhardt und Biermann. 